\title{
Evaluation of Heat-related IIIness Surveillance Based on Chief Complaint Data from New Jersey Hospital Emergency Rooms
}

\author{
Michael Berry ${ }^{1}$, Jerald Fagliano ${ }^{1}$, Stella Tsai ${ }^{* 1}$, Katharine McGreevy ${ }^{1}$, Andrew Walsh ${ }^{2}$ and \\ Teresa Hamby ${ }^{1}$
}

${ }^{1} \mathrm{NJDOH}$, Trenton, NJ, USA; ${ }^{2}$ Health Monitoring Systems, Inc., Pittsburgh, PA, USA

\section{Objective}

The purpose of this evaluation is to characterize the relationship between a patient's initial hospital emergency room chief complaint potentially related to a heat-related illness (HRI) with final primary and secondary ICD-9 diagnoses.

\section{Introduction}

The NJ syndromic surveillance system, EpiCenter, developed an algorithm to quantify HRI visits using chief complaint data. While heat advisories are released by the National Weather Service, an effective HRI algorithm could provide real-time health impact information that could be used to provide supplemental warnings to the public during a prolonged heat wave

\section{Methods}

Data on NJ hospital emergency room visits were evaluated using two data sources: 1) the EpiCenter syndromic surveillance system of emergency room visits; and 2) the Uniform Bill-Patient Summaries (UB) system containing diagnosis data on all hospital visits. Three years of data (2009-2011) were selected, for the time window of May 1 to September 30. The UB data used for matching with the EpiCenter data were limited to facilities participating in EpiCenter during the evaluation period. (EpiCenter facilities captured about 1/3 of all heat-related diagnoses in 2009 , increasing to about $2 / 3$ in 2011.) The ICD-9 codes of interest included 992.0-992.9 and external cause of injury codes E900.0 and E900.9. We evaluated the sensitivity and positive predictive value (PPV) of the EpiCenter algorithm in relation to the patients' eventual diagnoses coded in the UB data.

\section{Results}

During the 15 months of data examined, there were a total of 871 people identified with HRI visits based on the EpiCenter algorithm. Over the same time period in the same emergency room facilities, there were a total of 2,146 people with a primary or secondary HRI diagnosis in UB. The algorithm for the EpiCenter's HRI definition had a sensitivity of $16 \%(348 / 2,146)$ when any primary or secondary ICD or E-code matched; the PPV was $40 \%(348 / 871)$. When data during a major heat event (July 21-23, 2011) was examined separately, both sensitivity (23\%) and PPV (59\%) improved.

Graph 1 presents the 2011 daily number of HRI visits from EpiCenter data and the subset of UB data from facilities also reporting to EpiCenter. The pattern in the EpiCenter data tracked with the UB data for HRI visits and correctly identified several major episodes in 2011.

The major heat-related illness episode of July 2011 was selected to evaluate the non-matched EpiCenter and UB data. A total of 210 $(95 \%)$ of the non-matched UB cases were able to be matched to EpiCenter chief complaint data. The EpiCenter information displayed a diverse range of general complaints, including syncope, dizziness, weakness, and headache. Similarly, non-matched EpiCenter data were compared to UB data to examine diagnoses, and 22 (48\%) of the EpiCenter HRI cases were matched to UB diagnostic data. Diagnosis codes for these cases were for a variety of conditions classified under "general symptoms"; fluid balance disorders; asthma; diabetes; and unspecified hypertension.

\section{Conclusions}

The evaluation found that using chief complaint data to monitor HRI was relatively insensitive in comparison to the UB diagnosis codes, with a sensitivity of just over $16 \%$ for any UB HRI diagnosis. Sensitivity and PPV improved during a peak heat event.

The evaluation of the non-matched data (both EpiCenter and UB) provided little guidance for modifying the algorithm. While expanding the algorithm to include complaints such as syncope, dizziness, or weakness may capture a few more HRI cases, it would also likely result in a greater number of false positive cases (i.e., higher background noise).

Though not especially sensitive, EpiCenter data did identify all major episodes of HRI in 2011. The degree of correspondence indicates that the EpiCenter HRI algorithm provides a useful real-time gauge of the daily HRI trends.

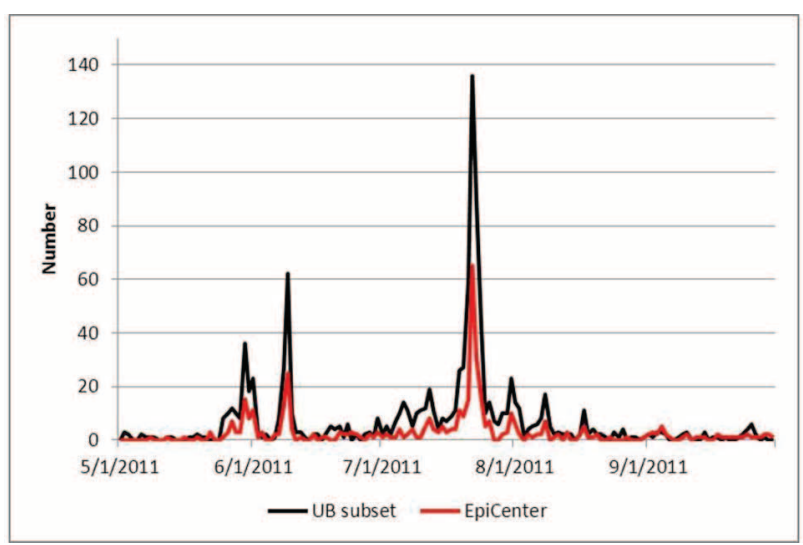

Graph 1. 2011 HRI visits identified by EpiCenter data and UB data subset for the same EpiCenter reporting facilities.

\section{Keywords}

Chief complaint; Heat Related Illness; UB data

\section{Acknowledgments}

This evaluation was supported in part by the Environmental Public Health Tracking Program, Centers for Disease Control and Prevention, Cooperative Agreement \#5U38EH000196.

\section{*Stella Tsai}

E-mail: stella.tsai@doh.state.nj.us 